\title{
Peculiarities of Matrix-Element Calculations with Few Coulomb Functions for Particles' Scattering Processes
}

\author{
Ochbadrakh Chuluunbaatar ${ }^{1,2, \star}, K_{\text {Konstantin Kouzakov }}^{3}$, and Yuri Popov ${ }^{4,1}$ \\ ${ }^{1}$ Joint Institute for Nuclear Research, Dubna, Moscow region 141980, Russia \\ ${ }^{2}$ Institue of Mathematics, National University of Mongolia, Ulan-Bator, Mongolia \\ ${ }^{3}$ Department of Nuclear Physics and Quantum Theory of Collisions, Faculty of Physics, Lomonosov Moscow \\ State University, Moscow 119991, Russia \\ ${ }^{4}$ Skobeltsyn Institute of Nuclear Physics, Lomonosov Moscow State University, Moscow 119991, Russia
}

\begin{abstract}
Specific features of the matrix-element calculations in the momentum-space representation are discussed for the single ionization of the He atom by fast proton impact in the case when the Coulomb interactions of all three charged fragments in the final state are taken into account. It is shown that a "soft" smoothing of the Coulomb singularity does not affect the accuracy of the calculations in a certain region of the smoothing parameter values.
\end{abstract}

\section{Introduction}

The computation of integrals containing Coulomb functions is typically encountered in the problems of scattering involving charged particles. A well-known example of such scattering problems is the ionization of an atomic target by electron or ion impact. In the last two decades, due to the remarkable progress of the cold target recoil ion momentum spectroscopy (COLTRIMS) [1], much interest has been directed toward the study of the single ionization of atomic targets by fast ions in the perturbative regime. Different theoretical approaches, in particular, those based on the plane wave first Born approximation (PWFBA), can be very robustly tested by comparing their predictions for the fully differential cross sections (FDCS) with the COLTRIMS data.

Currently there are two main theoretical approaches to the study of the ionization of a quantum target under the impact of a positive ion. These are the continuum distorted wave-eikonal initial state (CDW-EIS) method in the semiclassical and fully quantum formulations respectively $[2,3]$. In the semiclassical approach, one treats the projectile ion as a classical particle moving along a straight-line trajectory with an impact parameter $\rho$ and velocity $v_{p}$, which induces a time-dependent perturbation of the quantum target. In the quantum approach, one solves stationary quantum scattering problems for all particles involved in the ionization reaction.

It is to be mentioned that the CDW-EIS model involves three or more Coulomb functions or their asymptotic expressions in the matrix-element integration. They enter the descriptions of the interactions of the charged fragments both in the initial and in the final channels of the reaction. The matrix element itself can be written either in the real space or in the momentum space. There

\footnotetext{
^e-mail: chuka@jinr.ru
} 
are calculations in real-space representation with three and even four Coulomb functions (see, for instance, [4]). However, due to the complexity of the involved integrations, only simple trial wave functions of the target are employed. In the momentum space one has less three integrations due to the conservation laws but, at the same time, the Coulomb functions in the momentum-space representation have singularities with which one has to cope with and to perform correctly the regularization of the integrals.

In this work, we present calculations of the FDCS beyond the PWFBA theory, in order to explain the shift of the measured binary and recoil peaks in the scattering plane by a few degrees towards the incident proton direction with respect to the PWFBA predictions [5]. To this purpose we examine the well-known 3C (or Brauner-Briggs-Klar) model [6] and three different helium ground-state wave functions. Atomic units (a.u., $\hbar=e=m_{e}=1$ ) are used throughout unless otherwise specified.

\section{Theory}

Let us recall some well-known properties of a continuum Coulomb wave function. This function is a solution of the stationary Schrödinger equation and in the real-space representation it has the form

$$
\tilde{\phi}^{ \pm}(\vec{k}, \vec{r} ; Z)=e^{-\pi \eta / 2} \Gamma(1 \pm i \eta) e^{i \vec{k} \cdot \vec{r}}{ }_{1} F_{1}(\mp i \eta, 1 ; \pm i k r-i \vec{k} \cdot \vec{r}), \quad \tilde{\phi}^{-}(\vec{k}, \vec{r} ; Z)=\tilde{\phi}^{+*}(-\vec{k}, \vec{r} ; Z) .
$$

In (1), $\eta=Z / v, Z=Z_{1} Z_{2}$, where $Z_{1}$ and $Z_{2}$ are the charges of the colliding fragments, $v, \vec{k}$ and $\vec{r}$ are respectively the relative velocity, momentum and position of the colliding fragments, and ${ }_{1} F_{1}$ is the confluent hypergeometric function.

To calculate the Fourier transform of the Coulomb function one usually introduces a smoothing parameter $\lambda$, for example, as follows:

$$
\begin{aligned}
\phi^{ \pm}(\vec{k}, \vec{p} ; Z)= & \lim _{\lambda \rightarrow+0} \int d^{3} r e^{-\lambda r-i \vec{p} \cdot \vec{r}} \tilde{\phi}^{ \pm}(\vec{k}, \vec{r} ; Z)=8 \pi e^{-\pi \eta / 2} \Gamma(1 \pm i \eta) \\
& \times \lim _{\lambda \rightarrow+0}\left[\lambda(1 \pm i \eta) \frac{\left[(\lambda \mp i k)^{2}+p^{2}\right]^{ \pm i \eta}}{\left[(\vec{p}-\vec{k})^{2}+\lambda^{2}\right]^{2 \pm i \eta}} \mp i \eta(\lambda \mp i k) \frac{\left[(\lambda \mp i k)^{2}+p^{2}\right]^{-1 \pm i \eta}}{\left[(\vec{p}-\vec{k})^{2}+\lambda^{2}\right]^{1 \pm i \eta}}\right] .
\end{aligned}
$$

It is well known that in the limit $\lambda \rightarrow+0$ the integral of a product involving the first strongly singular term in the square brackets and any smooth function yields zero, and the second term is used as a wave function in the momentum space. The latter term is also singular that makes certain difficulties to deal with, a reason for which most authors prefer to work in the real-space representation.

Let us turn to this problem. For the sake of clarity, we start with some preliminary definitions. We designate the incident proton momentum by $\vec{p}_{i}$ and the final momentum by $\vec{p}_{s}$, the electron momentum by $\vec{k}_{e}$, the final ion momentum by $\vec{K}_{\text {ion }}$. The momentum transfer is $\vec{Q}=\vec{p}_{i}-\vec{p}_{s}=\vec{k}_{e}+\vec{K}_{\text {ion }}$. In the experiment for which the calculations have been carried out, $E_{p}=1 \mathrm{MeV}$, i.e. $v_{p}=\sqrt{2 E_{p} / m_{p}}=$ 6.35 a.u., $E_{e}=6.5 \mathrm{eV}$. From the energy conservation one obtains the $z$ component of the momentum transfer as $Q_{z}=\left(-\varepsilon_{0}^{\mathrm{He}}+\varepsilon_{0}^{\mathrm{He}^{+}}+E_{e}\right) / v_{p}=0.18$ a.u. (the $z$ axis is directed along the initial proton momentum $\left.\vec{p}_{i}\right)$. The transverse component is $Q_{\perp} \approx m_{p} v_{p} \theta_{s}=0.73$ a.u. $\left(\theta_{s}\right.$ is the proton scattering angle).

The general expression for the triple differential cross section (TDCS) is

$$
\frac{d^{3} \sigma}{d E_{e} d \Omega_{e} d \Omega_{s}}=k_{e} \frac{m_{p}^{2}}{(2 \pi)^{5}}\left|T_{\mathrm{fi}}\right|^{2} .
$$

The final state of the discussed reaction contains three charged fragments: the proton, electron, and $\mathrm{He}^{+}$ion. Below we consider a correlated 3C final-state wave function [6]. In this case the amplitude 
$T_{\mathrm{fi}}$ splits into the sum of three integrals, $T_{\mathrm{fi}}=A_{1}+A_{2}+A_{3}$, where

$$
\begin{aligned}
A_{1}= & 2 \sqrt{2} \int \frac{d^{3} p}{(2 \pi)^{3}} \phi^{-*}\left(\vec{v}_{p}-\vec{k}_{e}, \vec{p}+\vec{v}_{p}-\vec{k}_{e} ;-1\right) I\left(\vec{p}_{r}, \vec{Q}+\vec{p}_{r}-\vec{p} ; 1\right) G\left(\vec{k}_{e}, \vec{p}, 0\right), \\
A_{2}= & -\sqrt{2} \int \frac{d^{3} p}{(2 \pi)^{3}} I\left(\vec{v}_{p}-\vec{k}_{e}, \vec{p}+\vec{v}_{p}-\vec{k}_{e} ;-1\right) \phi^{-*}\left(\vec{p}_{r}, \vec{Q}+\vec{p}_{r}-\vec{p} ; 1\right) G\left(\overrightarrow{k_{e}}, \vec{p}, 0\right), \\
A_{3}= & -4 \pi \sqrt{2} \int \frac{d^{3} p_{1}}{(2 \pi)^{3}} \frac{d^{3} p_{2}}{(2 \pi)^{3} p_{2}^{2}} \phi^{-*}\left(\vec{v}_{p}-\vec{k}_{e}, \vec{p}_{1}+\vec{v}_{p}-\vec{k}_{e} ;-1\right) \\
& \times \phi^{-*}\left(\vec{p}_{r}, \vec{Q}+\vec{p}_{r}-\vec{p}_{1}-\vec{p}_{2} ; 1\right) G\left(\vec{k}_{e}, \vec{p}_{1}, \vec{p}_{2}\right) .
\end{aligned}
$$

In (4)-(6)

$$
\vec{p}_{r}=\left[\left(4 m_{p}+1\right) \vec{p}_{s}-m_{p} \vec{K}\right] /\left(5 m_{p}+1\right) \approx\left(4 m_{p} / 5\right) \vec{v}_{p}+(1 / 5) \vec{k}_{e}-\vec{Q},
$$

and also we have used the following notations:

$$
I(\vec{p}, \vec{q} ; Z)=\lim _{\lambda \rightarrow+0} \int \frac{d^{3} r}{r} e^{-\lambda r} \tilde{\phi}^{-*}(\vec{p}, \vec{r} ; Z) e^{i \vec{q} \cdot \vec{r}}=\lim _{\lambda \rightarrow+0} 4 \pi e^{-\pi \eta / 2} \Gamma(1+i \eta) \frac{\left[q^{2}-(p+i \lambda)^{2}\right]^{i \xi}}{\left[(\vec{q}-\vec{p})^{2}+\lambda^{2}\right]^{(1+i \xi)}},
$$

and

$$
G\left(\vec{k}, \vec{q}_{1}, \vec{q}_{2}\right)=\int d^{3} r_{1} d^{3} r_{2} \tilde{\phi}^{-*}\left(\vec{k}, \vec{r}_{1} ;-1\right) e^{i \vec{q}_{1} \cdot \vec{r}_{1}} \varphi_{0}^{\mathrm{He}^{+}}\left(\vec{r}_{2}\right) e^{i \vec{q}_{2} \cdot \vec{r}_{2}} \Phi_{0}^{\mathrm{He}}\left(\vec{r}_{1}, \vec{r}_{2}\right)
$$

For most of the He ground-state models the function $G\left(\vec{k}, \vec{q}_{1}, \vec{q}_{2}\right)$ is an analytical function or an integral of much lower dimension.

Three initial trial helium wave functions $\Phi_{0}^{\mathrm{He}}$ are employed: a weakly correlated RoothaanHartree-Fock (RHF) [7] function, $\varepsilon_{0}^{\mathrm{RHF}}=-2.8617$ a.u., a simple Silverman-Platas-Matsen (SPM) function [8] of the configuration interaction family, $\varepsilon_{0}^{\mathrm{SPM}}=-2.8952$ a.u., and a strongly correlated function (CF) [9] which explicitly depends on the $r_{12}$ distance between the electrons in helium, $\varepsilon_{0}^{\mathrm{CF}}=-2.903724$ a.u. The helium energy of the last function is very close to the experimental value, $\varepsilon_{0}^{\mathrm{He}}=-2.903724$ a.u.

\section{Results and discussion}

The purpose of the present study is to investigate the $\lambda$ dependence of the TDCS. In this regard it should be noted that the integrand functions in (4) and (5) in the vicinity of the weak singular points $\vec{p}=0$ and $\vec{p}-\vec{Q}=0$ at $\lambda \rightarrow 0+$ have the behaviors

$$
\frac{\lambda}{\left(\lambda^{2}+p^{2}\right)^{2}} \frac{1}{\left[\lambda^{2}+(\vec{p}-\vec{Q})^{2}\right]}
$$

and

$$
\begin{aligned}
& \int_{\Omega_{0}} d^{3} p \frac{\lambda}{\left(\lambda^{2}+p^{2}\right)^{2}}=2 \pi\left(\arctan \left(\frac{P}{\lambda}\right)-\frac{\lambda P}{\lambda^{2}+P^{2}}\right) \rightarrow \pi^{2}, \quad p \leq P \ll 1, \\
& \int_{\Omega_{Q}} d^{3} p \frac{1}{\left(\lambda^{2}+(\vec{p}-\vec{Q})^{2}\right)}=4 \pi\left(P-\lambda \arctan \left(\frac{P}{\lambda}\right)\right) \rightarrow 4 \pi P, \quad|\vec{p}-\vec{Q}| \leq P \ll 1 .
\end{aligned}
$$

The integrand function of the term (6) in the vicinity of the weak singular points $\vec{p}_{1}=0$ and $\vec{p}_{1}+\vec{p}_{2}-$ $\vec{Q}=0$ at $\lambda \rightarrow 0+$ has the behavior

$$
\frac{\lambda}{\left(\lambda^{2}+p_{1}^{2}\right)^{2}} \frac{1}{p_{2}^{2}} \frac{\lambda}{\left(\lambda^{2}+\left(\vec{p}_{1}+\vec{p}_{2}-\vec{Q}\right)^{2}\right)^{2}} .
$$



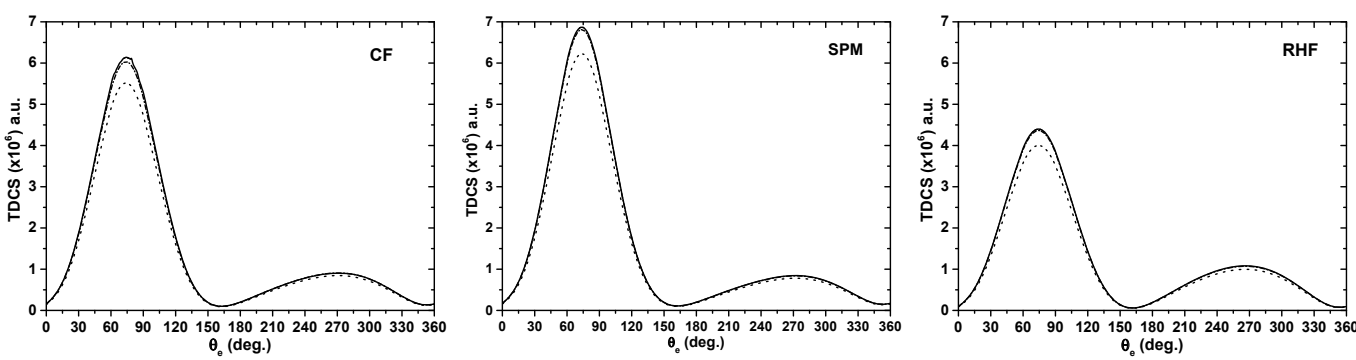

Figure 1. TDCS in coplanar geometry using different trial helium ground-state wave functions: CF (left), SPM (middle), and RHF (right). The solid line corresponds to $\lambda=10^{-4}$, the dash-dotted line to $\lambda=10^{-3}$, and the dashed line to $\lambda=10^{-2}$.

It means that the integral (6) has a singular point $\vec{p}_{2}=-\vec{p}_{1}+\vec{Q}$ in each direction of $\vec{p}_{1}$ and is numerically unstable. After using special transformation for each trial function, RHF, SPM and CF, the term (6) has the same singularities as the terms (4) and (5). Finally, the 3D, 4D and 7D integrals (depending on the trial functions) are calculated numerically using the Fortran code Cuhre [10].

We use (2) and keep the parameter $\lambda$ small but finite, namely in the range $10^{-4}-10^{-2}$. Our numerical approach exhibits quite good agreement with different test analytical calculations using the function (1). However, when $\lambda$ falls below $10^{-4}$, problems with the accuracy arise. At the same time, within the domain indicated above we observe good accuracy and stability of calculations. Numerical results for TDCS using different trial helium ground-state wave functions are shown in Fig. 1. Thus, the present study demonstrates the existence of a finite domain of smoothing-parameter values where the numerical calculations exhibit very good convergence and stability.

\section{Acknowledgments}

The present research benefited from computational resources of the Central Information and Computer Complex and the HybriLIT heterogeneous computing cluster of the Joint Institute for Nuclear Research. Yu. P. is grateful to Russian Foundation for Basic Research (RFBR) for the financial support under the grant No. 16-02-00049-a. O. Ch. acknowledges support from the Hulubei-Meshcheryakov program JINR-Romania.

\section{References}

[1] J. Ullrich et al, J. Phys. B 30, 2917 (1997); R. Dörner et al, Phys. Rep. 330, 95 (2000); J. Ullrich et al, Rep. Prog. Phys. 66, 1463 (2003)

[2] Dz. Belkic, R. Gayet, and A. Salin, Phys. Rep. 56, 281 (1979)

[3] D. S. F. Crothers and L. J. Dube, Adv. At. Mol. Opt. Phys. 30, 287 (1993)

[4] A. Mondal, Ch. Mandal, and M. Purkait, EPJD 70, 16 (2016)

[5] H. Gassert et al., Phys. Rev. Lett. 116, 073201 (2016)

[6] M. Brauner, J. S. Briggs, and H. Klar, J. Phys. B: At. Mol. Opt. Phys. 22, 2265 (1989)

[7] E. Clementi and C. Roetti, At. Data Nucl. Data Tables 14, 177 (1974)

[8] J. N. Silvermann, O. Platas, and F. A. Matsen, J. Chem. Phys. 32, 1402 (1960)

[9] O. Chuluunbaatar et al, Phys. Rev. A 74, 014703 (2006)

[10] http://www.feynarts.de/cuba/ 